\title{
Long-term follow-up of pain and quality-of-life scores after laparoscopic adhesiolysis
}

Published online: 24 July 2004

(C) Springer-Verlag Berlin / Heidelberg 2004

\begin{abstract}
There is increasing evidence that laparoscopic adhesiolysis improves chronic pelvic pain. We performed a long-term review of women after laparoscopic adhesiolysis over the past 4 years. Patients were excluded from the study if they had additional pathology such as endometriosis or required additional procedures other than adhesiolysis. Umbilical insertion of Verress' needle and primary trocar was used except when the patient had had a previous midline laparotomy, in which case Palmer's point was used for entry. Adhesions were divided using Metzenbaum scissors with haemostasis using suction irrigation achieved with a Surgiflex $\mathrm{R}$ Wave ${ }^{\mathrm{TM}}$ suction irrigation system with BICAP ${ }^{\mathrm{TM}}$ bipolar diathermy probe (ACMITM, USA). Hydroflotation with heparinised saline or $4 \%$ icodextrin was used to reduce adhesion recurrence. Patients were sent a postal questionnaire and contacted by telephone. Visual analogue scales were used to record pain scores for dysmenorrhoea (in those women who still had a uterus), dyspareunia, dyschezia and chronic daily pain. An EQ-5D questionnaire was also enclosed to assess quality of life. One hundred and forty-three procedures were identified between September 1998 and July 2002. Having excluded those with additional pathology that required treatment, 90 were eligible for the study. Seventy-six replies were obtained; seven patients had moved away. Sixty-nine replies were analysed. Fifty-one (74\%) reported some improvement in their symptoms [12 (17\%) pain completely gone, 26 (38\%) greatly improved, 13 (19\%) a little better]. Patients still had significant pain [scores out of 100 for dysmenorrhoea (45), dyspareunia (28), dyschezia (28) and daily pain (29)]. Overall, quality of life was still lower than national averages (self-rated health status mean $=67.0$ vs. $82.34, P<0.05$, weighted health state index $=0.67$ vs. $0.85, P<0.05$ ), except in the good responders (pain gone or greatly improved, for whom quality of life returned to normal). There was no
\end{abstract}

\footnotetext{
J. English $(\bowtie) \cdot J$. Ford · T. Giannopoulos Department of Obstetrics and Gynaecology, Worthing Hospital,

Lyndhurst Road Worthing, West Sussex, BN11 2DH, UK

e-mail: James.English@btinternet.com
}

difference in pain scores, response and quality of life between women who had had their surgery more than 24 months earlier and those who had had surgery more recently. We have found a good response to adhesiolysis, which is comparable with other studies. A good response is associated with a normal quality of life and appears to be long standing.

Keywords Laparoscopy · Adhesiolysis · Pain · Quality of life

\section{Introduction}

The importance of adhesions as a cause of abdominal and pelvic pain is increasingly recognised [1, 2]. Post-surgical adhesions in the abdomen and pelvis are frequently identified in women with a history of chronic pain persisting for more than 6 months [3], but the merit of lysis of these adhesions remains uncertain. Evidence has been mounting that adhesiolysis is beneficial in terms of pain $[4,5,6,7]$, but some doubt recently has been cast on this, suggesting that any perceived benefit may largely be in terms of placebo effect [8]. In view of the reported complications associated with surgery in the presence of adhesions [8], the potential benefits of laparoscopic adhesiolysis need to be more clearly defined. This is brought into greater relief by the realisation that one is effectively using surgery to treat a condition that is often secondary to surgery and that any therapeutic benefit will depend not just on the adequacy of the lysis of the adhesions, but perhaps even more on their failure to recur. Yet, success or failure, at least from the patient's viewpoint, is governed less by the recurrence of any adhesions than by the response in terms of pain and the improvement in quality of life. It has been considered that laparoscopic surgery has been less prone than open surgery to result in adhesion formation [9]. However, recent evidence casts some doubt on this, making it less than certain. The purpose of this study is to assess patient response to laparoscopic adhesiolysis, to look at their current pain scores and 
quality of life and to report any perioperative or longer term complications.

\section{Materials and methods}

This study involves the reporting of a continuous case series of women with a history of pelvic or abdominal pain for more than 6 months who were seen in the pelvic pain clinic and were offered a laparoscopy. These women were then included in the study if they were found to have adhesions, but were excluded from the study if they had additional pathology such as endometriosis or required additional procedures other than adhesiolysis. Umbilical insertion of Verress' needle and primary trocar was used except when the patient had had a previous midline laparotomy, in which case Palmer's point was routinely used for entry. Routine bowel preparation with Picolax was employed in those patients known to have dense colonic adhesions or who had symptoms suggestive of such. Adhesions were divided using Metzenbaum scissors with haemostasis using suction irrigation achieved with a Surgiflex R WaveTM suction irrigation system with BICAPTM bipolar diathermy probe (ACMITM, USA). Hydroflotation with heparinised saline or $4 \%$ icodextrin was used to reduce adhesion recurrence. Patients were sent a postal questionnaire and contacted by telephone. Visual analogue scales were used to record pain scores for dysmenorrhoea (in those women who still had a uterus), dyspareunia, dyschezia and chronic daily pain. An EQ-5D questionnaire was also enclosed to assess quality of life.

\section{Results}

One hundred and forty-three procedures were identified between September 1998 and July 2002. Having excluded those with additional pathology that required treatment, 90 were eligible for the study. Seventy-six replies were obtained; seven patients had moved away. Sixty-nine replies were analysed. Fifty-one (74\%) reported some improvement in their symptoms [2 $(17 \%)$ pain completely gone, $26(38 \%)$ greatly improved, $13(19 \%)$ a little better]. Patients still had significant pain [scores out of 100 for dysmenorrhoea (45), dyspareunia (28), dyschezia (28) and daily pain (29); Table 1]. Overall, quality of life was still lower than national averages (self-rated health status mean $=67.0$ vs. $82.34, P<0.05$, weighted health state index $=0.67$ vs. $0.85, P<0.05$ ), except in the good responders (pain gone or greatly improved), for whom quality of life returned to normal (Table 2). There was no difference in pain scores, response and quality of life between women who had had their surgery more than 24 months earlier and those who had had surgery more

Table 1 Response to surgery in terms of subjective pain

\begin{tabular}{lccc}
\hline & Overall & $\begin{array}{c}\text { Under } \\
24 \text { months }\end{array}$ & $\begin{array}{c}\text { Over } \\
24 \text { months }\end{array}$ \\
\hline Pain gone & & 3 & 9 \\
Greatly improved & 12 & 15 & 11 \\
A little better & 13 & 6 & 7 \\
No change & 12 & 8 & 4 \\
Worse & 6 & 3 & 3 \\
Total & 69 & 35 & 34 \\
\hline
\end{tabular}

Table 2 Postoperative quality of life scores

\begin{tabular}{lcll}
\hline & Overall & $\begin{array}{l}\text { Under } \\
24 \text { months }\end{array}$ & $\begin{array}{l}\text { Over } \\
24 \text { months }\end{array}$ \\
\hline $\begin{array}{l}\text { Health self-rated } \\
\text { Population mean }\end{array}$ & 67 & 63 & 62 \\
Mean weighted & & 85 & 85 \\
$\begin{array}{l}\text { Health state index } \\
\text { Population mean }\end{array}$ & 0.67 & 0.61 & 0.74 \\
\hline
\end{tabular}

Table 3 Postoperative pain scores

\begin{tabular}{llll}
\hline & Overall & $\begin{array}{l}\text { Under } \\
24 \text { months }\end{array}$ & $\begin{array}{l}\text { Over } \\
24 \text { months }\end{array}$ \\
\hline Dysmenorrhea & 45 & 48 & 42 \\
Dyspareunia & 28 & 28 & 28 \\
Dyschezia & 28 & 29 & 26 \\
Chronic pain & 29 & 32 & 26 \\
\hline
\end{tabular}

recently (Table 3 ). There were no significant complications.

\section{Discussion}

Our series shows a generally favourable response to laparoscopic adhesiolysis and supports the findings of previous work $[4,5,7]$, adding to the growing evidence to support the therapeutic merit of adhesiolysis in the treatment of abdominal and pelvic pain. Recent work by Swank et al. [8] has suggested that much of the benefit derived from laparoscopic adhesiolysis is a placebo effect. However, this latter study ignored the work of Demco [10], who demonstrated by pain-mapping that pain is not necessarily perceived at the site of pathology; secondly, and perhaps more importantly, it did not utilise adhesion barriers, thus potentially increasing the risk of recurrence following adhesiolysis. In this regard, a placebo effect could only have been confirmed by means of a second-look laparoscopy to demonstrate a significant reduction in the adhesions in the adhesiolysis group despite a lack of improvement in pain scores. While laparoscopy has previously been demonstrated to have a significant placebo effect [11], any benefit derived has been shown to decrease markedly over time. Our study found no difference in benefit for those who had had their surgery been 2 and 4 years previously and those whose surgery had been less than 2 years previously, suggesting that a significant placebo effect is unlikely.

Certain authors $[12,13,14]$ have argued that adhesions either do not cause pain or, even if they do, then it is only when they result in bowel obstruction. This contention is not supported by Demco [10], who was able to reproduce pain by probing adhesions during wake laparoscopy and by the evidence of the growth of sensory nerves into adhesion bands [15]. None of the patients in the present study had presented with bowel obstruction, but all with pain. Swank et al. [8] argued the case for performing an incomplete adhesiolysis if the surgery became difficult 
with increased risks of haemorrhage and viscus perforation. Yet, perhaps, it is precisely this group of patients who most require radical and complete surgery, not only to correct the resultant anatomical abnormalities created by the adhesions, but also to identify underlying pathology, such as cul de sac endometriosis, which may otherwise fail to be identified by incomplete dissection. While other authors have suggested that laparoscopic adhesiolysis is associated with very high rates of bowel injury of up to $25 \%$, and that this should be a reason for reluctance to perform the operation $[8,16]$, there was no bowel injury in the current series, suggesting in line with other series [17] that high complication rates are not inevitable. All of the patients in our series had postoperative flotation of the pelvic and abdominal organs, some with heparinised saline and most with 4\% icodextrin (Adept, Shire). Our study did not have the power to demonstrate any difference in outcome.

In conclusion, we have found a good response to adhesiolysis that is comparable to other studies. A good response is associated with a normal quality of life and appears to be long standing.

\section{References}

1. Lower AM, Hawthorne RJS, Ellis H (2000) The impact of adhesions on hospital readmissions over 10 years after 8,489 open gynaecological operations: an assessment from the Surgical and Clinical Adhesions Research Study. Br J Obstet Gynaecol 107:855-862

2. Parker M, Ellis H, Moran BJ, Thompson JN, Wilson MS (2001) Postoperative adhesions: 10-year follow-up of 12,584 patients undergoing lower abdominal surgery. Dis Colon Rectum 44:822-830

3. Giannopoulos T, Ford J, Dimitriou E, English J (2003) Clinical analysis of primary laparoscopies for pelvic pain (abstract). Rev Gynaecol Pract
4. Sutton C, MacDonald R (1990) Laser laparoscopic adhesiolysis. J Gynaecol Surg 6:155

5. Nezhat FR, Crystal RA, Nezhat CH, Nezhat CR (2000) Laparoscopic adhesiolysis and relief of chronic abdominal pain. JSLS 4:281-285

6. Cueto-Rozon R, Bordea A, Barrat C, Gillion JF, Catheline JM, Fagniez PL, Champault (2000) Is laparoscopic treatment of adhesions a valid approach for postoperative pain? Giornale de Chiurgia 21:433-437

7. Steege JF, Stout AL (1991) Resolution of chronic pelvic pain after laparoscopic lysis of adhesions. Am J Obstet Gynecol $165: 278$

8. Swank DJ, Swank-Bordewijk SCG, Hop WCJ, van Erp WFM, Janssen IMC, Bonjer HJ, Jeekel J (2003) Laparoscopic adhesiolysis in patients with chronic abdominal pain: a blinded randomised controlled multi-centre trial. Lancet 361:12471251

9. Maier DB, Nulsen JC, Klock A, Luciano AA (1992) Laser laparoscopy versus laparotomy in lysis of pelvic adhesions. J Reprod Med 37:965

10. Demco LA (2000) Pain referral patterns in the pelvis. J Am Assoc Gyn Lap 7:181-183

11. Sutton CJG, Ewen SP, Whitelaw N, Haines P (1994) Preospective, randomised, double-blind, randomised controlled trial of laser laparoscopy in the treatment of pelvic pain associated with minimal, mild and moderate endometriosis. Fertil Steril 62:696-700

12. Ellis H, Moran BJ, Thompson NJ et al (1999) Adhesion-related hospital readmissions after abdominal and pelvic surgery: a retrospective cohort study. Lancet 353:1476-1480

13. Ellis H (1997) The clinical significance of adhesions: focus on intestinal obstruction. Eur J Surg [Suppl] 577:5-9

14. Alexander-Williams J (1987) Do adhesions cause pain? Br Med J 294:659

15. Sulaiman H, Gabella G, Davis C, Mutsaers SE, Boulos P, Laurent GJ, Herrick SE (2001) Presence and distribution of sensory nerve fibres in human peritoneal adhesions. Ann Surg 234:256-261

16. van der Krabben AA, Dijkstra FR, Nieuwenhuijzen M, Reijnen MMPJ (2000) Morbidity and mortality of inadvertant enterotomy during adhesiotomy. Brit J Surg 87:467-471

17. Carbajo Caballero MA, Martin del Olmo JC, Blanco JI, Martin F, Cuesta MT (2001) Therapeutic value of adhesiolysis. Surg Endoscopy 15:102-103 